\title{
In situ monitoring of pit nucleation and growth at iron passive oxide layer using combined scanning force - scanning electrochemical microscopy
}

\author{
Javier Izquierdo, ${ }^{[a]}$ Alexander Eifert, ${ }^{[b]}$ Christine Kranz, ${ }^{[b]}$ and Ricardo M. Souto ${ }^{[a]}$
}

\begin{abstract}
Generation of single corrosion pits and in situ monitoring of pit growth on iron exposed to $0.5 \mathrm{M} \mathrm{NaCl}$ solution was achieved using combined atomic force - scanning electrochemical microscopy (AFMSECM). Pits as small as $2.7 \mu \mathrm{m}$ in diameter were formed at arbitrary locations on the substrate by local generation of highly concentrated nitric acid in the vicinity of the AFM-SECM probe. Addition of nitrite ions to the environment, which act as corrosion inhibitors for iron, ensures passivation of the metal, and hinders metal corrosion despite exposure to the chloride-containing media. Localized acidification was achieved by oxidizing nitrite ions at the probe. Acidification in combination with the high chloride content in the solution leads to a local rapid attack at the surface and pit generation below the AFM-SECM probe. Besides improved spatial resolution and precise control of the pit nucleation site, combined AFM-SECM allows simultaneous imaging of the generated pits by the AFM tip.
\end{abstract}

Keywords: localized corrosion, single pit nucleation, iron, combined AFM-SECM, imaging

\section{Introduction}

Metallic materials are usually employed in a state of passivation with an oxide layer formed on their surface acting as a barrier to ionic migration effectively separating the underlying metal from its environment. ${ }^{[1]}$ As a result, the release of metal ions from the substrate into the electrolytic phase is greatly hindered, and the material is regarded to be stable for sufficiently long service periods. Unfortunately, passivity breakdown and localized pitting corrosion are common phenomena to many materials in chloride solutions. In that event, though most of the material remains passive, effectively experiencing very slow corrosion rates, pits propagate very rapidly penetrating into the bulk of the material. Failure and even catastrophic destruction of the material often occurs as result of such penetration, ${ }^{[1]}$ which is the motivation for the significant research efforts investigating the causes and mechanisms of pitting. Yet, no unifying model has been proposed until now. As pitting is in its initial stages a highly localized process, forming "holes" or cavities in the order of micrometers or below, the detection and monitoring of the early stages of pitting is impeded. ${ }^{[2]}$ Though pit nucleation can often be correlated to heterogeneities in the material, pits can also be nucleated on homogeneous metallic surfaces, producing random distributions as a result. ${ }^{\left[{ }^{3]}\right.}$ Significant progress in the knowledge of the origins of corrosion has been gathered from electrochemical measurements in the micrometer range, in order to greatly reduce the number of pitting events simultaneously occurring on the investigated section of the substrate. Two main technical approaches were thus attempted, either reduction in the size of the metal sample undergoing passivity breakdown by using microelectrodes, ${ }^{[4,5]}$ or the reduction of the exposed area by miniaturizing significantly the electrochemical cell in order to characterize individual features on the sample. ${ }^{[6,7]}$ In the first case, the recorded electrochemical signal averages the response of the whole exposed miniaturized substrate, though its small dimensions allow the events related to individual breakdown processes to be resolved above the background response of the remaining passive surface. ${ }^{[4]}$ The stochastic nature of pitting could be described in terms of size and distribution of events, ${ }^{[3,4]}$ and single transients could be analyzed in order to identify separate pitting stages. ${ }^{[3,4,8]}$ Yet, the lack of spatial resolution and imaging required the sequential use of surface characterization methods to correlate kinetic information to pit shape and pit distribution, ${ }^{\left[{ }^{[-11]}\right.}$ as well as the occurrence of topographic changes inside and around the nucleated pits. ${ }^{[4,11]}$ Microscopic methods including high resolution in situ atomic force microscopy (AFM) have been applied for topographic characterization of substrates undergoing corrosion but only pits already in the propagation stage have been imaged. ${ }^{[12-15]}$ Alternatively, microelectrochemical cells enabled the individual characterization of the electrochemical response of smaller features on the substrates such as inclusions or grain boundaries and thus correlating the 
electrochemical response to the corrosion behaviour in those sites. ${ }^{[7]}$ Yet, the combined effect of those features operating in a real corrosion process, usually related to microgalvanic effects and potential field distributions, would be missing from those experiments.

Therefore, the inherently localized nature of the pitting process requires methods capable to gain information about the generation and evolution of the pit formation in the micrometer range with high spatial resolution, preferably in real time. In this regard, scanning electrochemical microscopy (SECM) is highly attractive for corrosion studies, and various experimental procedures have been developed for studying corrosion phenomena. Identification of precursor sites for the nucleation of pitting corrosion, ${ }^{[16-20]}$ and the visualization of metastable pitting ${ }^{[21]}$ were achieved using SECM. Despite the differences in measuring procedures employed, these investigations have in common that they only report spatial fluctuations in the passive current measured on the investigated materials related to passivity breakdown, either leading to metastable pitting and subsequent repassivation, ${ }^{[21]}$ or to further propagation into the stable pitting regime. ${ }^{[16-20]}$ Hence, monitoring was only possible once the pit was nucleated. Even more SECM studies have effectively focused on the identification of the corrosion products from pits already in their propagation stage. ${ }^{[22-26]}$

A different approach to characterize pitting corrosion using SECM consists in the controlled generation of corrosion pits due to the localized nature of the technique. This can be achieved either by electrically forcing a direct current to flow between the passive substrate and the tip (known as direct mode SECM), ${ }^{[27]}$ or by locally generating an aggressive species that promotes the onset of pitting. ${ }^{[28-33]}$ In the first case, the application of a strong electric field between the sample and the microelectrode positioned in its close proximity produces a localized breakdown of the poorly conductive oxide layer of dimensions comparable to those of the active surface of the microelectrode. ${ }^{[27]}$ In fact, a low ionic strength of the electrolyte results in a strong electric field developed between the sample and the tip to operate on a small portion of the surface. The requisite of a critical minimum chloride concentration in the electrolyte to trigger oxide breakdown for pit generation using a silver chloride coated microelectrode prevents this method from being applicable to more stable materials such as i.e., stainless steel. As reported in literature, artificial pits on $316 \mathrm{~L}$ stainless steel were generated using SECM in $10 \mathrm{M}$ chloride concentration ${ }^{[35]}$, whereas pits were also produced in direct mode SECM in a solution containing only $1 \mathrm{mM} \mathrm{KCl} .{ }^{[27]}$ Unfortunately, though small defects are effectively produced in the passive oxide layer using this method, the breakdown mechanism involved does not correlate to actual pitting mechanisms because the process is not originated by the local interaction of aggressive ions such as chloride.

Several procedures have been developed in combination with SECM for the local release of chloride ions in the vicinity of the site selected for the nucleation of the corrosion pit. Local chloride release has been achieved by the reduction of chloride precursors dissolved in the electrolyte solution at the SECM probe, ${ }^{[28,29]}$ or the reduction of a silver chloride layer deposited on silver microelectrodes. ${ }^{[30-33]}$ The nucleation of single pits requires a small electrolyte volume contained between the tip and the sample to reach a critical chloride concentration that is characteristic for each material. Thus, the generation of pits on iron was achieved by both approaches releasing chloride ions, ${ }^{[29-33]}$ but they could only be nucleated on stainless steel using a silver/silver chloride microelectrode because higher concentrations of the anion were produced ${ }^{[34]} A$ more recent improvement consists in the replacement of the chlorinated silver UME by a microcapillary for continuous injection of a chloride-containing solution of adequate concentration. ${ }^{[7,11,34]}$ Finally, electrolysis of water has also been used to alter locally the $\mathrm{pH}$-value and induce alkaline or acid attacks in the vicinity of susceptible surfaces ${ }^{[35]}$ of dimensions limited only by the size of the ultramicroelectrodes (UMEs). Smaller defects were produced in this work compared to those described before using chloride ions because the local corrosive attack corresponded to chemical dissolution of the alumina layers. The dissolution process occurred by shifting the $\mathrm{pH}$ of the electrolyte beyond the stability range of the precipitated surface layer, and not by the initiation of a pitting corrosion reaction.

The achievable spot size is critically limited by the size of the UME required to build a sufficiently high local concentration of the aggressive ions. Furthermore, subsequent analysis should not be constrained to the characterization of the electrochemical nature (composition and activity) of the electrolyte in the close vicinity of the formed pit, but also to investigate the topography and roughness changes as a result of the localized breakdown, though distinction between both contributions is not straightforward in SECM. This difficulty may be eliminated with the combination of scanning probe microscopy techniques such as AFM-SECM, providing the excellent topographic information of AFM while microelectrochemical experiments are performed. Combining AFM with SECM was demonstrated fabricating a modified AFM probe with a conductive frame or ring electrode around the insulating AFM tip. ${ }^{[36,37]}$ Alternatively, a cantilever-shaped disc microelectrode insulated with Parylene $C$ can also be employed as the AFM tip, as it was demonstrated by Davoodi et al. ${ }^{[38]}$ The latter probe design was used in corrosion studies for the characterization of the role of intermetallic particles present in heterogeneous alloys towards the onset of localized corrosion. ${ }^{[38,39]}$

Recently, a novel concept for local pit initiation using combined AFM-SECM was introduced. The precursor of the aggressive species responsible for the breakdown of the passive oxide layers was locally generated at an AFM-SECM probe ${ }^{[40]}$ By taking advantage of this principle and the smaller electroactive area integrated in AFM-SECM probes, a more localized chemical field can be produced, ample to deliver locally a sufficient concentration of the active species for the generation of a single pit on a passivated metal surface. Whereas in 
the initial work the basic concept was demonstrated, this contribution focuses more on the mechanistic and experimental aspects of the process as to develop an efficient methodology for locally inducing pitting corrosion at well-defined spots on an iron surface using combined AFM-SECM using different experimental approaches.

\section{Nucleation of corrosion pits in nitrite solution}

Visualization of pit nucleation and propagation using either SECM or AFM is difficult due to the stochastic nature of pit distribution and the small dimensions of pitting at early stages. Hence, single pit generation induced by the actual chemical species involved in passivity breakdown without the application of an external potential to the sample is highly attractive. SECM operating in the tip generator-substrate collection mode can generate the critical local concentration of the chemical species involved in the initiation of pitting corrosion, though restricted by the dimensions of the microelectrode active area required to build a critical concentration of the aggressive ions. Due to a significantly smaller dimension of the AFM tip-integrated electrode, the chemical field required to generate a sufficient concentration of aggressive electrolyte within the gap between the AFM tipintegrated electrode and the substrate surface gets more focused.

Instead of the local release of chloride ions, a local $\mathrm{pH}$ modification can induce the break down of the passive layer in chloride containing solution. A chloride-containing solution with a sufficiently high concentration for pit initiation was considered as the test solution, but the solution $\mathrm{pH}$ was shifted into the stability domain of iron oxides according to the corresponding Pourbaix diagram. ${ }^{[41]}$ Accordingly, $0.5 \mathrm{M}$ chloride concentration was selected in this work, which is significantly higher than the critical value of $0.1 \mathrm{M}$ established by Vivier and coworkers for iron. ${ }^{[32]}$ In this context, the function of the AFM-SECM probe towards pit generation is the local generation of a sufficient acidic environment for the aggressive attack of this chloride concentration to occur. The oxidation of nitrite salts at the AFM tip-integrated electrode is hereby a very interesting approach as this oxidation reaction results in nitrate ions and the release of protons as by-product according to reaction (1):[42,43]

$$
\mathrm{NO}_{2}^{-}+\mathrm{H}_{2} \mathrm{O} \rightarrow \mathrm{NO}_{3}^{-}+2 \mathrm{H}^{+}+2 \mathrm{e}^{-}(1)
$$

A second advantageous effect of nitrite ions derives from the corrosion inhibitor characteristics of this species for iron, contributing to the protection of the remaining metal surface from corrosion stabilizing its passive state. The most accepted mechanism to describe corrosion inhibition of iron by nitrite ions is based on their ability to oxidize both chemically adsorbed and diffusing iron(II) species released by the corroding metal. ${ }^{[45-47]}$ As a result, iron(III) is formed that undergoes hydrolysis more easily, therefore leading to poorly soluble ferric hydroxides and oxides, which precipitate on the iron surface thus hindering corrosion. It has been proposed that maghemite $\mathrm{Y}-\mathrm{Fe}_{2} \mathrm{O}_{3}$ is the main product formed through reaction (2) when passive conditions are established, ${ }^{[44,45]}$ whereas $\mathrm{FeOOH}$ phases are rapidly produced following reaction (3) when pitting corrosion occurs. ${ }^{[4,46]}$

$$
\mathrm{Fe}^{2+}+2 \mathrm{OH}^{-}+2 \mathrm{NO}_{2}^{-} \rightarrow 2 \mathrm{NO}+\mathrm{y}^{-}-\mathrm{Fe}_{2} \mathrm{O}_{3}+\mathrm{H}_{2} \mathrm{O}
$$

$$
4 \mathrm{Fe}(\mathrm{OH})_{2(\mathrm{aq})}+2 \mathrm{NO}_{2}^{-} \rightarrow \mathrm{N}_{2} \mathrm{O}+4 \mathrm{FeOOH}_{(\mathrm{aq})}+2 \mathrm{OH}^{-}+\mathrm{H}_{2} \mathrm{O}
$$

In addition, it has been suggested that nitrite ions compete for adsorption at the active sites present on the iron surface in chloride-containing solutions, effectively enhancing the pitting corrosion resistance of the metal. ${ }^{[47]}$ The oxidation of the adsorbed nitrite ions may lead to the generation of oxide anions through halfreaction (4) ${ }^{[45]}$ As result, the solubility product $K s$ for iron oxide is immediately exceeded by iron cations produced in the corrosion process leading to precipitation.

$$
2 \mathrm{NO}_{2}^{-}+4 \mathrm{e}^{-} \rightarrow 2 \mathrm{~N}_{2} \mathrm{O}+3 \mathrm{O}^{2-}
$$

In summary, applying a potential to the AFM tip-integrated gold microelectrode, preferably within the diffusionlimited oxidation range of nitrite ion oxidation, generates locally a high concentration of protons from nitrite anions. Since a fairly high concentration of chloride anions is already present in solution, a highly localized fast corrosion process should be induced on the iron substrate in the vicinity of the combined AFM-SECM tip. Meanwhile the remaining exposed area of the metal substrate is protected by the inhibiting effect of the sodium nitrite, which behaves as corrosion inhibitor for iron surfaces in $0.5 \mathrm{M}$ chloride solution when the nitrite/chloride ratio is $1: 5 .{ }^{[46]}$

\section{Results and Discussion}




\section{Electrochemical behavior of gold microelectrodes in nitrite solution}

Initial experiments investigating the generation of nitrate and proton ions were performed by linear sweep voltammetry recorded at a gold disc microelectrode in $0.5 \mathrm{M}$ sodium chloride both in the presence and in the absence of sodium nitrite. The monitored current responses are shown in Figure 1a. The black curve shows the current response recorded in nitrite-free solution, where the only visible signal corresponds to water oxidation at potential values higher than $+1.0 \mathrm{~V}_{\mathrm{Ag} / \mathrm{AgCl} / \mathrm{KCl} \text { (sat.). }}$. When nitrite is present in solution, the current profile shows an additional oxidation process at less positive potential values, approximately at $+0.7 \mathrm{~V}_{\mathrm{Ag} / \mathrm{AgCl} / \mathrm{KCl} \text { (sat.), which }}$ clearly indicates the onset of half-reaction (1). Therefore, a constant potential of $+0.95 \mathrm{VAg} / \mathrm{AgCl} / \mathrm{KCl}$ (sat.), sufficiently anodic to ensure nitrite oxidation but preventing water electrolysis, was selected to investigate the formation of nitric acid at the gold surface.

The time evolution of the current flowing through the gold microelectrode at a constant polarization of +0.95

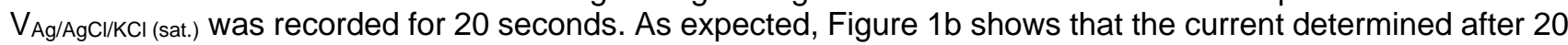
seconds was around $0.11 \mu \mathrm{A}$ when the solution contained nitrite salt, whereas the current measured in the sodium chloride background solution was over one magnitude order smaller. This current range involves, considering that the measured faradaic response comes solely from nitrite oxidation to nitrate and proton generation (cf. equation (1)), a nitrate generation of $5.7 \times 10^{-13} \mathrm{~mol} \mathrm{~s}^{-1}$, and twice this amount, $1.14 \times 10^{-12} \mathrm{~mol} \mathrm{~s}^{-}$ ${ }_{1}^{1}$ for proton production. Though a more precise determination of these local concentration increments would require accurate modelling of the actual probe-substrate geometry, estimations should be sufficient at this stage. By considering a $10 \mu \mathrm{m}$ diameter hemisphere surrounding the active gold micro disc as the base of a cylinder in which these species are dissolved, the concentration increase for nitrates and protons will be 2.18 and 4.35 mol L-1 $\mathrm{s}^{-1}$, respectively, if diffusion of these species towards bulk solution is neglected due to geometrical hindrance.

\section{Generation of single corrosion pits on iron}

Iron surfaces were locally attacked and AFM images were recorded before and after pitting. Figure 2 presents topography images of an iron surface before (Fig. 2a) and after (Fig. 2b) inducing local pitting. Pitting formation in the presented experiment was induced by scanning a $3 \times 3 \mu \mathrm{m}^{2}$ area of the surface at a scan speed of 3.84 $\mu \mathrm{m} \mathrm{s}^{-1}$ while applying $+0.95 \mathrm{VAg} / \mathrm{AgCl} / \mathrm{KCl}$ (sat.) to the AFM tip-integrated electrode. The site for pit generation was chosen at the center of a larger scan area given in Figure 2a. The topography after pitting is shown in Figure $2 \mathrm{~b}$, which clearly shows a circular hole that corresponds to the nucleation of a single pit and remains apparently unchanged after 2 hours, as line scans presented in Figure 2c demonstrate. Data of the line scans are raw data (no post-levelling procedure was performed), in order to monitor the actual height differences. Only shifts in $Z$ coordinates were performed to set the value to zero at the minimum $Z$ measured. Since the initial width of the formed pit was ca. $8.5 \mu \mathrm{m}$, its size was close to the dimensions of the thin layer cell formed below the moving AFM-SECM probe. It should be noted that the actual electrode of the probe is determined by the $1.07 \mu \mathrm{m}$ length of the square $A u$ electrode plus the additional $1 \mu \mathrm{m}$ of the insulation layer surrounding the frame electrode (exemplary AFM-SECM probe shown in micrograph in Figure $2 \mathrm{e}$ ). Correspondingly, for a $3 \times 3 \mu \mathrm{m}^{2}$ scan, the surface area covered by the probe has an approximate diameter of $7.1 \mu \mathrm{m}$. Thus, confinement of the aggressive species below the integrated electrode is the main factor responsible for the corroding attack to the passive oxide film, though some widening of the concentration field due to convection and diffusion of the generated aggressive species cannot be discarded and may be responsible for the slightly larger dimensions of the pit. Yet, the concentration changes are (mostly) constrained to the small electrolyte volume determined by the size of the AFM-SECM probe and the length of the thorn of the cantilever (ca. $460 \mathrm{~nm}$ ).

A series of scan lines were subsequently recorded over a single generated pit in order to monitor its evolution in the chloride + nitrite containing medium. The probe was moved along the $X$ axis in fixed $Y$ position where maximum depth was observed in the previous map. A selection of these line scans is given in Figure 2c. No major changes were observed between the line scans with the elapse of time apart from small variations in the shape of the height profiles and a slight diminution in noise level. It is proposed that rapid repassivation of the surface must have occurred, due to the generation of $\mathrm{FeOOH}$ phases, ${ }^{[44,46]}$ shortly after pit generation. This feature was probably induced due to the diffusion of fresh nitrite content into the area after the attack was stopped and the tip was moved far from the attack site to initiate the AFM imaging step. Furthermore, the $\mathrm{FeOOH}$ phases would be converted into maghemite $\mathrm{y}-\mathrm{Fe}_{2} \mathrm{O}_{3}$ as the experimental series proceed, resulting in a smoother surface and the above mentioned noise level decline. Moreover, ongoing pit formation should lead to a progressing growth of the hole with time, yet the evolution in depth and width as displayed in Figure $2 \mathrm{~d}$ did not change over time, supporting the hypothesis of a heterogeneous growth of an oxide layer, which is apparently more pronounced inside the hole.

Combined AFM-SECM also enables the electrochemical characterization towards the constituents of the medium in close proximity to the surface. It is in principle possible to determine the spatial distribution of dissolved metal from the nucleated pit in the event of growth into the propagation stage. Metal dissolution releases iron(II) ions that can be monitored from their electro-oxidation to iron(III) at the AFM-SECM probe 
operating in the substrate generation - tip collection (SG-TC) mode. ${ }^{[21,22,48]}$ It should be noted that any formation of iron(II) species during the pit generation step would pass unnoticed because of the potential applied for nitrite

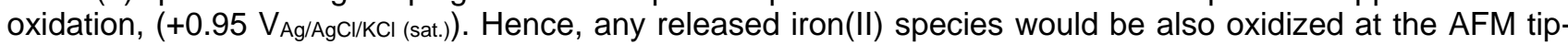
integrated electrode and the recorded current would reflect both reactions. Thus, following pit induction and recording of AFM image in Figure $3 a$ over a larger area centred around the generated pit, the tip potential was shifted from +0.95 to $+0.55 \mathrm{~V}_{\mathrm{Ag} / \mathrm{AgCl} / \mathrm{KCl} \text { (sat.). }}$. This potential value is positive enough to oxidize iron(II) to iron(III), but not high enough to form nitrate anions. Then, both the progress of local corrosion and the distribution of iron(II) were sequentially imaged by scanning a $3 \times 3 \mu \mathrm{m}^{2}$ area at the centre of the previously imaged larger region. However, no local iron(II) concentration could be detected at the AFM-SECM probe polarized at +0.55

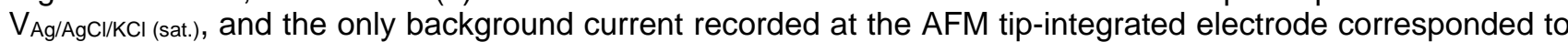
the background current related to the passive regime of the metal (not shown). On the other hand, oxygen must be consumed during the corrosion process in the cathodic half-reaction, and therefore its concentration must become depleted in the solution adjacent to the cathodic areas of the surface. Oxygen depletion can also be monitored by SECM. ${ }^{[22,49]}$ Thus, the tip potential was switched to $-0.55 \mathrm{~V}$ Ag/AgC//KCl (sat.), negative enough to promote the electro-reduction of oxygen, and a larger $30 \times 30 \mu \mathrm{m}^{2}$ area scan was imaged around the same region imaged in Figure 3a. The resulting AFM map is shown in Figure 3b. Again, only a homogeneously distributed faradaic current was registered this time, and no evidence of localized oxygen consumption could be detected.

No evidences of pit propagation were thus observed, though the topography recorded over this large area revealed some changes following the induction of pit corrosion. Figure $3 a$ shows the initial topography scan when no localized pitting had been induced, and Figure 3b shows the topography of the same area after performing the pit-induction experiments described above. In this case, instead of a hole, the occurrence of some precipitates was encountered, corresponding to insoluble corrosion products. This result may be explained in terms of the electrochemical process occurring in the small confined volume below the AFM-SECM tip, which forms a "leaking" thin layer cell. The electrochemical nature of this thin electrolyte volume is actively modified by the reactions occurring at the AFM tip-integrated gold electrode while scanning a $3 \times 3 \mu \mathrm{m}^{2}$ area at such close distance. Moreover, diffusion of the inhibitor into, and/or diffusion of ferrous species out of this volume into the solution may be attenuated as long as the tip is not retracted from the surface. As a result, the oxidation of iron(II) at the AFM tip-integrated electrode leads to the local accumulation of ferric cations, that form ferric hydroxides due to to the hydrolysis of the soluble metal ions. These hydroxides may precipitate producing a deposit of corrosion products. Reduction of oxygen during subsequent scans may also contribute to this process, since alkalization occurs during its reduction at the tip, and surface passivation is favoured in alkaline environments. Yet, the hydroxyl formation might have only a minor effect as most of the iron(II) species were already oxidized at the electrode by the time the scan started with the electrode biased at $E_{\text {AFM-SECM tip }}=-0.55$ $\mathrm{V}_{\mathrm{Ag} / \mathrm{AgCl} / \mathrm{KCl} \text { (sat.) }}$ and hence no further precipitation of iron(III) hydroxides occurred. The diameter of the observed deposit is $5.15 \mu \mathrm{m}$, comparable to what would be expected when only the above-mentioned dimensions of the active area and scan for attack are considered. This feature supports that the discussed diffusion limitation is one of the predominant motivations for the formation of the precipitate, since larger protrusions would otherwise be observed.

The reproducibility of pit formation and the influence of size of the scan area were investigated in a new series of experiments in which the area was sequentially reduced. A freshly polished iron sample was imaged by AFM with a scan area of $50 \times 50 \mu \mathrm{m}^{2}$, prior a pitting experiment induced by scanning a $3 \times 3 \mu \mathrm{m}^{2}$ area with the AFMSECM probe biased at $E_{\mathrm{AFM}-\mathrm{SECM} \text { tip }}=+0.95 \mathrm{~V}_{\mathrm{Ag} / \mathrm{AgCl} / \mathrm{KCl} \text { (sat.) }}$ was performed (i.e., identical experimental conditions as in the experiment depicted in Figure $2 b$ ). Subsequently, the procedure was repeated at a different location within the initially imaged $50 \times 50 \mu \mathrm{m}^{2}$ area, though decreasing the scan size down to $1 \times 1 \mu \mathrm{m}^{2}$. Next, the AFMSECM probe was positioned at three other locations while maintaining the same tip potential, but now pit formation was induced without scanning the tip at different durations of the polarization experiments. Results from these two sets of experiments, identified as scanning and stationary procedures are depicted in Figure 4. AFM images recorded before and after the indicated sequence are displayed in Figures $4 \mathrm{a}$ and $4 \mathrm{~b}$, respectively. It is clearly visible that the longer the scan duration, and therefore the more aggressive the conditions, the deeper and the wider the resulting pit was. For the three experiments performed in the stationary procedure, zoomed views of the corresponding pits are shown in Figure 4c, with their characteristic widths and depths are listed in Table 1. The smallest pit was produced using the stationary mode with 20 seconds polarization time as shown in the corresponding deflection image (Figure 4d). Zoomed views are also given in Figure 4e. This operating condition seems to be the lowest operational limit attainable to produce the aggressiveness required to generate a pit.

The influence of the duration of the exposure of the sample in the test solution was also investigated. It was anticipated that longer exposure times resulted in the generation of larger pits, but the same effect might arise from an increase in the chloride concentration in the small electrochemical cell due to solvent evaporation during the course of the experiment. Therefore, a similar series of experiments was performed on the same sample one hour after the previous one was completed using the scanning mode, but employing sub-micrometer scan sizes of $0.75 \times 0.75 \mu \mathrm{m}^{2}, 0.5 \times 0.5 \mu \mathrm{m}^{2}$, and $0.25 \times 0.25 \mu \mathrm{m}^{2}$, respectively. The topography obtained after these 
experiments is shown in Figure $4 \mathrm{f}$ revealing that the pits generated by scanning sub-micron sized areas were comparable in size to those formed during the $3 \times 3 \mu \mathrm{m}^{2}$ and the $1 \times 1 \mu \mathrm{m}^{2}$ scans. Table 1 also gives the width and depth of the generated pits using smaller scan sizes, supporting this hypothesis. Actually, corrosion pits formed at longer exposure times are typically deeper and wider. For pits formed in the second series, less time and smaller scan sizes of the attacks were necessary to produce pits with similar size as those resulting from larger scans when immersed for a shorter time. For instance, the $0.75 \times 0.75 \mu \mathrm{m}^{2}$ attack produced during the second experimental series can be compared to that previously obtained with $3 \times 3 \mu \mathrm{m}^{2}$ area scan, because they resulted in similar pit widths (7290 and $8100 \mathrm{~nm})$ and depths ( $834 \mathrm{~nm}$ and $754 \mathrm{~nm})$. Similar results were obtained for pits generated by scanning $0.25 \times 0.25 \mu \mathrm{m}^{2}$ and $1 \times 1 \mu \mathrm{m}^{2}$ with the AFM-SECM probe performed on the same sample without electrolyte exchange.

Diffusion of nitrite ions and fast repassivation are considered to occur once the tip is moved away from the location of the attack, as derived from the results shown in Figure 2. To corroborate these observations, another strategy for pit generation was tested. Now the potential was applied to the AFM-SECM probe in short pulses of $0.5 \mathrm{~s}$ duration, followed by relaxation time sequences at $0 \mathrm{~V}_{\mathrm{Ag} / \mathrm{AgCl} / \mathrm{KCl} \text { (sat.) }}$ of the same duration, every time using the scanning mode though with different scan dimensions. In order to compare the results obtained using either constant or pulsed polarization of the AFM tip-integrated electrode, the same rate and scan dimensions were employed though at different locations of the surface. The AFM topography of the iron surface after the application of both constant and pulsed polarization for pit generation are depicted in Figure 5 . The pits generated under constant polarization of the probe are located on left half of the imaged area, whereas the right side displays the experiments using pulsed polarization. No evidence of local degradation was found at the locations affected by pulsed polarization, and pits were only generated when applying a constant potential to the probe. Hence, we can conclude that continuous inhibitor consumption is required, accompanied by local formation of the aggressive species, for the aggressive attack to produce breakdown of the surface oxide layer. The dimensions of the pits generated under the constant potential regime are consistent with the scan sizes attained during local pitting, as it was expected. Furthermore, when a series of AFM scans are obtained over one such pit once it was nucleated, the role of repassivation could be considered. Thus, as time elapses after formation of the induced pit, both pit volume and depth decrease as depicted in Figure 6, which reveals the growth of the passivation layer.

Finally, it should be considered that the aggressive media produced during the pitting experiment may have an adverse effect on the stability of the AFM tip-integrated gold electrode. In fact, mixtures of $\mathrm{HNO}_{3}$ and $\mathrm{HCl}$ are commonly employed for metallurgical purposes during gold extraction, and they are also employed for etching of gold surfaces.[50] Therefore, the active area of the probe was determined recording CVs in hexaaminruthenium(III) solution at the beginning and at the end of each experiment to ensure that the active electrode area remained basically unaltered. In addition, the applied potential at the probe was also swept in the sodium nitrite test solution employed during measurements. Results for both experiments are shown in Figure 7. The cyclic voltammograms depicted in Figure 7a were recorded in the hexaaminruthenium(III) solution before and after completing one experimental series for local pit nucleation of 5 pits with dimensions ranging from 7 to $12 \mu \mathrm{m}$ in diameter. The stability of the AFM tip-integrated gold electrode is revealed by the well matching voltammograms shown in Figure $7 \mathrm{a}$, which indicates that no significant etching occurred at the gold surface. The formation of nitric acid is indeed observed during nitrite oxidation at positive potentials, as shown in Figure 7b.

\section{Conclusions}

The combined AFM-SECM technique has been applied to induce localized corrosion on otherwise passive surfaces. The size of the obtained single pit was investigated in respect to the influence of the experimental parameters. The use of nitrite ions leads to a passivation of the iron surface. The localized oxidation of nitrite at the AFM-SECM probes in a tip generation - substrate collection type experiment enables the simultaneous generation of the pitting precursor species, and the local removal of the passivating agent. This procedure effectively leads to the nucleation of single corrosion pits at the surface of iron in a chloride-containing solution. Pit formation only occurs as long as the inhibiting species is consumed at the AFM-SECM probe and hence prevents the restoration of inhibitive conditions in the selected area, where an aggressive environment has being created, permitting the local attack to progress. The presence of an inorganic corrosion inhibitor in bulk solution that diffuses into the area of pit formation, promotes repassivation once pitting induction has stopped (no further potential applied to the probe). Further electrochemical modification of the surrounding media may induce the precipitation of corrosion products giving rise to the observation of topographic protrusions around the pits.

Different experimental conditions for nitrite oxidation at the AFM tip-integrated electrode for the induction of sufficiently large single pits have been explored. It was found that the pitting generation strategy involving scanning the AFM-SECM probe, while producing the reactive species allowed greater control of pit dimensions 
for comparable immersion times. Furthermore, the continuous application of a sufficiently positive potential to the AFM-SECM probe is required to generate conditions aggressive enough for the nucleated pit to grow in depth. Besides, using AFM-SECM technique, dimensions of the generated pits can be as small as $1.2-2.7$ $\mu \mathrm{m}$ in width. These ranges are beyond the limits of the previously reported pit induction procedures using conventional SECM, ${ }^{[28-33]}$ due to the larger electroactive areas of conventional microelectrodes and the greater ability of the integrated AFM-SECM electrodes to chemically focus the electrogenerated aggressive species under the probe.

It was also shown that certain operating conditions may lead to fast accumulation of corrosion products, which precipitate inside the defect and may eventually protrude from the surface.

\section{Experimental Section}

\section{Reagents and samples}

All solutions were prepared using ultra pure water (Elga water system, conductivity $18.0 \mathrm{M} \Omega \mathrm{cm}$ (Elga Labwater; VWS Deutschland $\mathrm{GmbH}$, Celle, Germany) and chemicals of analytical grade. All experiments were carried out at ambient temperature (approximately $20^{\circ} \mathrm{C}$ ).

Pure $99.5 \%$ iron sheets of $1 \mathrm{~mm}$ thickness supplied by Goodfellow (Cambridge, UK) were employed as substrate material. They were cut into $2.5 \times 2.5 \mathrm{~cm}^{2}$ square specimens, then ground with silicon carbide papers of increasing grit size up to 4000 , and subsequently mechanically polished with alumina micropolish suspension (0.05 $\mu \mathrm{m}$ particle size) in deionized water until a mirror-like surface was obtained. The polished sample was thoroughly rinsed with high-purity water, immersed in ethanol and sonicated for 20 minutes in an ultrasonic bath for cleaning, and finally dried under argon gas stream.

\section{Instrumentation}

A 5500 atomic force microscope from Keysight Technologies (Chandler, AZ, USA) equipped, with a 90 um scanner was used for combined measurements. For all measurements, the Keysight SECM module consisting of a picostat potentiostat, an electrochemical AFM liquid cell and a specialized nose cone with built-in operation amplifier and all the necessary connections for SECM operation was used. The iron substrate was carefully mounted at the bottom of the electrochemical AFM liquid cell but left disconnected. Hence, the substrate was always kept at its spontaneous open circuit potential (OCP). The exposed surface area was circular shaped and approximately $1.75 \mathrm{~cm}$ in diameter. The total volume of electrolyte was approx. $1 \mathrm{~mL}$. A ring-shaped counter electrode made of platinum, and a chlorinated silver wire operating as pseudo-reference electrode completed the three-electrode arrangement with the AFM-SECM probe as working electrode. The potential of the Ag/AgCl wire pseudo-reference electrode was $+48 \mathrm{mV}$ vs. $\mathrm{Ag} / \mathrm{AgCl} / \mathrm{KCl}$ (sat.) reference electrode in the test solution, consisting of $0.5 \mathrm{M} \mathrm{NaCl}+0.1 \mathrm{M} \mathrm{NaNO}_{2}$, which is in good agreement with the theoretical value predicted by the Nernst equation considering the $0.5 \mathrm{M}$ chloride concentration used during experiments. For the sake of simplicity, all potential values given in this work are referred to the $\mathrm{Ag} / \mathrm{AgCl} / \mathrm{KCl}$ (sat.) reference electrode.

The fabrication of the AFM-SECM probes has been described elsewhere. ${ }^{[36,37]}$ In brief, a bare silicon nitride AFM cantilever is coated with gold as electrode layer. Consecutively, mixed silicon nitride/silicon oxide layers were deposited via chemical vapor deposition (CVD) to insulate electrically the metallized AFM cantilever. Afterwards, focused ion beam (FIB) milling (Quanta 3D FEG, FEI Company, Eindhoven, NL) is employed for precisely exposing a frame-shaped electrode and re-shaping the very end of the insulating AFM tip to form the thorn for high-resolution topography measurement. The individual milling steps were monitored with scanning electron microscopy (SEM), which enables determining the actual electrode size and AFM tip length and curvature of the tip apex. Finally, the electric contact to the contact pad of the probe was made with a Tefloninsulated wire and silver epoxy glue. The AFM-SECM probe used for the experiments had a square gold ring with $1.07 \mu \mathrm{m}$ in edge length (gold layer thickness: $100 \mathrm{~nm}$ ), and a re-shaped AFM tip of $0.46 \mu \mathrm{m}$ in height. AFMSECM probes were characterized in deoxygenated $10 \mathrm{mM}$ potassium hexaaminruthenium(III) solutions with 0.1 $\mathrm{M} \mathrm{KCl}$ as supporting electrolyte.

In a first step, the electrochemical reactions related to nitrite oxidation in chloride-containing media at a gold microelectrode $(10 \mu \mathrm{m}$ in diameter) were characterized using a $\mathrm{CH}-660 \mathrm{~A}$ potentiostat $(\mathrm{CH}$-Instruments, Austin, $\mathrm{TX}, \mathrm{USA}$ ). A platinum counter electrode and $\mathrm{Ag} / \mathrm{AgCl} / \mathrm{KCl}$ (sat.) reference electrode completed the cell, which contained around $3 \mathrm{~mL}$ of solution. The solution was naturally aerated and contained $0.5 \mathrm{M} \mathrm{NaCl}+x \mathrm{M} \mathrm{NaNO}$. The values of $x$ were 0 and 0.1 .

\section{Experimental procedures}

Three different operation procedures for inducing local pitting using AFM-SECM in contact mode were tested, with the goal to determine the smallest pit size that could be achieved and imaged at this stage. Nitrite oxidation was performed by applying a constant potential to the AFM tip-integrated gold electrode, EUME $=+0.95$

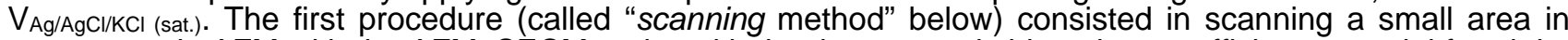
contact mode AFM with the AFM-SECM probe with the tip constantly biased at a sufficient potential for nitrite diffusion-limited oxidation. In this manner, various scan sizes ranging from $0.25 \times 0.25 \mu \mathrm{m}^{2}$ to $3 \times 3 \mu \mathrm{m}^{2}$ were 
performed using a scan rate of $3.84 \mu \mathrm{m} \mathrm{s}^{-1}$. A second procedure, termed "stationary method", was performed by applying the positive potential to the tip while the scanning was stopped and the AFM tip remained at a certain fixed position in contact with the substrate. For both procedures, the result of pitting on the substrate was imaged by in situ AFM, choosing an increased scan area around the positions of the initial pit formation. A third experimental series consisted of applying a sequence of short positive potential pulses followed by a relaxation time of the same duration while scanning again small areas, similarly to the previously described "scanning method". However, this third approach did not result in visible corrosion pits this time by using potential pulses of $0.5 \mathrm{~s}$ duration. In all experiments, the formed pits were always characterized by AFM immediately after completing the potential programme for corrosion induction using the same AFM-SECM probe in the same solution.

\section{Acknowledgements}

A grant awarded to J.I. by the Deutscher Akademischer Austauschdienst (Berlin, Germany) to conduct a research stay at the University of UIm is gratefully acknowledged. The Focused Ion Beam Center UUIm supported by FEI Company (Eindhoven, Netherlands), the German Science Foundation (INST40/385-F1UG), and the Struktur- und Innovationsfonds Baden-Württemberg are acknowledged for supporting this study. R.M.S. acknowledges support by the Spanish Ministry of Economy and Competitiveness (MINECO, Madrid) and the European Regional Development Fund, under grant CTQ2012-36787.

\section{References}

[1] G.T. Burstein in Corrosion. Volume 1: Metal/Environment Reactions (Eds.: L.L. Shreir, R.A. Jarman, G.T. Burstein), $3^{\text {rd }}$. edition, Butterworth-Heinemann, Oxford, 1994, pp. 1:118-1:150.

[2] G.T. Burstein, C. Liu, R.M. Souto, S.P. Vines, Corros. Eng. Sci. Technol. 2004, 39, 25-30.

[3] G.T. Burstein, R.M. Souto, Electrochim. Acta 1995, 40, 1881-1888.

[4] P.C. Pistorius, G.T. Burstein, Phil. Trans. R. Soc. Lond. A 1992, 341, 531-559.

[5] R.K. Gupta, N.L. Sukiman, M.K. Cavanaugh, B.R.W. Hinton, C.R. Hutchinson, N. Birbilis, Electrochim. Acta 2012, 66, 245-254

[6] T. Suter, H. Böhni, Electrochim. Acta 1997, 42, 3275-3280.

[7] V. Vignal, H. Krawiec, O. Heintz, R. Oltra, Electrochim. Acta 2007, 52, 4994-5001.

[8] G.S. Frankel, J. Electrochem. Soc. 1998, 145, 2186-2198.

[9] M.H. Moayed, R.C. Newman, J. Electrochem. Soc. 2006, 153, B330-B335.

[10] A.M. Zimer, E.C. Rios, L.H. Mascaro, E.C. Pereira, Electrochem. Commun. 2011, 13, 1484-1487.

[11] N. Aouina, F. Balbaud-Célérier, F. Huet, S. Joiret, H. Perrot, F. Rouillard, V. Vivier, Electrochim. Acta 2013, 104, 274-281.

[12] R.E. Williford, C.F. Windisch Jr, R.H. Jones, Mater. Sci. Eng. A 2000, 288, 54-60.

[13] F.A. Martin, C. Bataillon, J. Cousty, Corros. Sci. 2008, 50, 84-92.

[14] Y. Li, R. Hu, J. Wang, Y. Huang, C.-J. Lin, Electrochim. Acta 2009, 54, 7134-7140.

[15] V. Maurice, P. Marcus, Electrochim. Acta 2012, 84, 129-138.

[16] N. Casillas, S. Charlebois, W.H. Smyrl, H.S. White, J. Electrochem. Soc. 1994, 141, 636-642.

[17] Y.Y. Zhu, D.E. Williams, J. Electrochem. Soc. 1997, 144, L43-L45.

[18] L.F. Garfias-Mesias, M. Alodan, P.I. James, W.H. Smyrl, J. Electrochem. Soc. 1998, 145, $2005-2010$

[19] B.B. Katemann, C.G. Inchauspe, P.A. Castro, A. Schulte, E.J. Calvo, W. Schuhmann, Electrochim. Acta 2003, 48, 1115-1121.

[20] M.A. Malik, P.J. Kulesza, Anal. Chem. 2007, 79, 3996-4005.

[21] Y. González-García, G.T. Burstein, S. González, R.M. Souto, Electrochem. Commun. 2004, 6, 637-642.

[22] A.C. Bastos, A.M. Simões, S. González, Y. González-García, R.M. Souto, Electrochem. Commun. 2004, 6, 1212-1215.

[23] T.E. Lister, P.J. Pinhero, Anal. Chem. 2005, 77, 2601-2607.

[24] Y. Yin, L. Niu, M. Lu, W. Guo, S. Chen, Appl. Surf. Sci. 2009, 255, 9193-9199.

[25] C.F. Dong, H. Luo, K. Xiao, X.G. Li, Y.F. Cheng, J. Mater. Eng. Perform. 21 (2012) 406-410.

[26] J. Izquierdo, L. Martín-Ruíz, B.M. Fernández-Pérez, L. Fernández-Mérida, J.J. Santana, R.M. Souto, Electrochim. Acta 2014, 134, 167-175.

[27] K. Eckhard, M. Etienne, A. Schulte, W. Schuhmann, Electrochem. Commun. 2007, 9, 1793-1797.

[28] D.O. Wipf, Coll. Surf. A: Phys. Eng. Asp. 1994, 93, 251-261.

[29] J.W. Still, D.O. Wipf, J. Electrochem. Soc. 1997, 144, 2657-2665.

[30] K. Fushimi, K. Azumi, M. Seo, J. Electrochem. Soc. 2000, 147, 552-557.

[31] K. Fushimi, M. Seo, J. Electrochem. Soc. 2001, 148, B450-B456.

[32] C. Gabrielli, S. Joiret, M. Keddam, H. Perrot, N. Portail, P. Rousseau, V. Vivier, J. Electrochem. Soc. 2006, 153, B68-B74.

[33] C. Gabrielli, S. Joiret, M. Keddam, H. Perrot, N. Portail, P. Rousseau, V. Vivier, Electrochim. Acta 2007, 52. 7706-7714.

[34] N. Aouina, F. Balbaud-Célérier, F. Huet, S. Joiret, H. Perrot, F. Rouillard, V. Vivier, Electrochim. Acta 2011, $56,8589-8596$. 
[35] D. Battistel, S. Daniele, R. Gerbasi, M.A. Baldo, Thin Solid Films 2010, 518, 3625-3631.

[36] C. Kranz, G. Friedbacher, B. Mizaikoff, A. Lugstein, J. Smoliner, E. Bertagnolli, Anal. Chem. 2001, 73, 2491-2500.

[37] H. Shin, P.J. Hesketh, B. Mizaikoff, C. Kranz, Sensors and Actuators B: Chem. 2008, 134, $488-495$.

[38] A. Davoodi, J. Pan, C. Leygraf, S. Norgren, Electrochem. Solid-State Lett. 2005, 8, B21-B24.

[39] A. Davoodi, J. Pan, C. Leygraf, S. Norgren, Electrochim. Acta 2007, 52, 7697-7705.

[40] J. Izquierdo, A. Eifert, R.M. Souto, C. Kranz, Electrochem. Commun. 2015, 51, 15-18.

[41] M. Pourbaix, Atlas of Electrochemical Equilibria in Aqueous Solutions, Pergamon/CEBELCOR, Oxford, 1966, pp. 308-310.

[42] J. Ufheil. C. Heß. K. Borawarth. J. Heinze. Phvs. Chem. Chem. Phvs. 2005. 7. 3185-3190.

[43] V. Radtke, C. He $\beta$, R.M. Souto, J. Heinze, Z. Phys. Chem. 2006, 220, 393-406.

[44] L. Dhouibi, Ph. Refait, E. Triki, J.M.R. Génin, J. Mater. Sci. 2006, 41, 4928-4936.

[45] M. Reffass, R. Sabot, M. Jeannin, C. Berziou, Ph. Refait, Electrochim. Acta 2007, 52, 7599-7606.

[46] M.B. Valcarce, M. Vazquez, Mater. Chem. Phys. 2009, 115, 313-321.

[47] Y.M. Tang, Y. Zuo, X.H. Zhao, Corros. Sci. 2008, 50, 989-994.

[48] M. Zhao, Z. Qian, R. Qin, J. Yu, Y. Wang, L. Niu, Corros. Eng. Sci. Technol. 2013, 48, $270-275$.

[49] R.M. Souto, L. Fernández-Mérida, S. González, Electroanalysis 2009, 21, 2640-2646.

[50] D.A. Scott, Ancient Metals: Microstructure and Metallurgy, Vol. 1: Principles and Practice, Conservation Science Press, Los Angeles, 2010, p. 187.

Table 1. Dimensions of the pits shown in Figure 4. Pits were generated using either scanning (scan.) or stationary (stat.) modes at the indicated times while the iron surface was immersed in $0.5 \mathrm{M} \mathrm{NaCl}+0.1 \mathrm{M} \mathrm{NaNO}_{2}$ under open circuit conditions

\begin{tabular}{lccc}
\hline & Depth $/ \mathrm{nm}$ & Width $/ \mathrm{nm}$ & Immersion time / min \\
\hline scan. $3 \times 3 \mu \mathrm{m}^{2}$ & 754 & 8100 & 103 \\
\hline scan. $1 \times 1 \mu \mathrm{m}^{2}$ & 283 & 4850 & 117 \\
\hline scan. $0.75 \times 0.75 \mu \mathrm{m}^{2}$ & 834 & 7290 & 180 \\
\hline scan. $0.5 \times 5 \mu \mathrm{m}^{2}$ & 601 & 6070 & 184 \\
\hline scan. $0.25 \times 0.25 \mu \mathrm{m}^{2}$ & 235.4 & 4080 & 186 \\
\hline stat. (duration $=90 \mathrm{~s})$ & 85.4 & 3330 & 122 \\
\hline stat. (duration $=50 \mathrm{~s})$ & 143 & 2730 & 124 \\
\hline stat. (duration $=20 \mathrm{~s})$ & 80.1 & 1464 & 125 \\
\hline
\end{tabular}
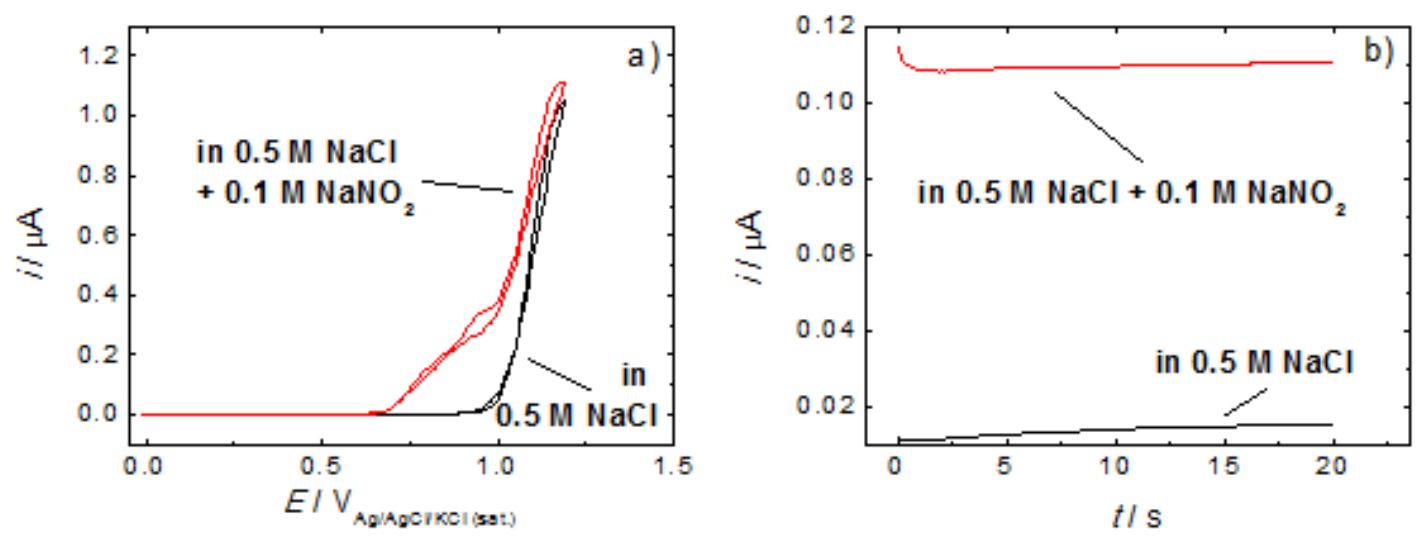

Figure 1. Current responses recorded at the $10 \mu \mathrm{m}$ diameter gold microdisc electrode in $0.5 \mathrm{M} \mathrm{NaCl}+x \mathrm{M}$ $\mathrm{NaNO}_{2}(x=0,0.1)$. (a) Linear sweep voltammetry between 0 and $+1.2 \mathrm{~V} \mathrm{vs.} \mathrm{Ag} / \mathrm{AgCl}$ (sat.); (b) constant potential measurement at $+0.95 \mathrm{~V}$ vs. $\mathrm{Ag} / \mathrm{AgCl}$ (sat.). 

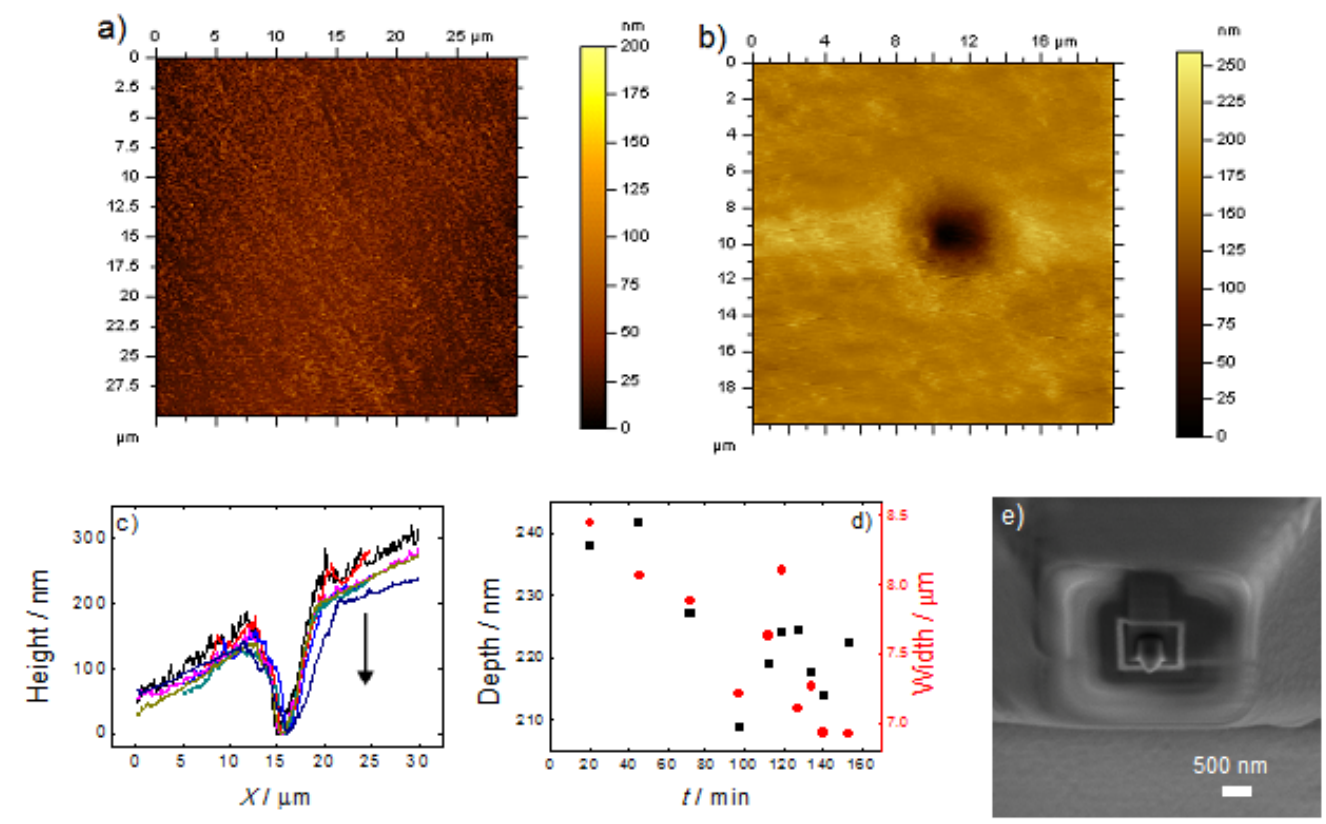

Figure 2. AFM topographies of the iron surface in $0.5 \mathrm{M} \mathrm{NaCl}+0.1 \mathrm{M} \mathrm{NaNO}_{2}$ at open circuit conditions (a) before and $(b-d)$ after generating a single pit at the center of the scanned area under study. Single pit evolution is represented in terms of: (c) line scans taken from a series of AFM images during pit formation by selecting the $Y$ coordinates where maximum pit depth was observed (arrow indicates longer times), and (d) plot of the depths and widths of the generated pits as a function of the times employed in their generation.

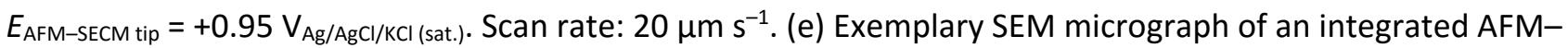
SECM probe.
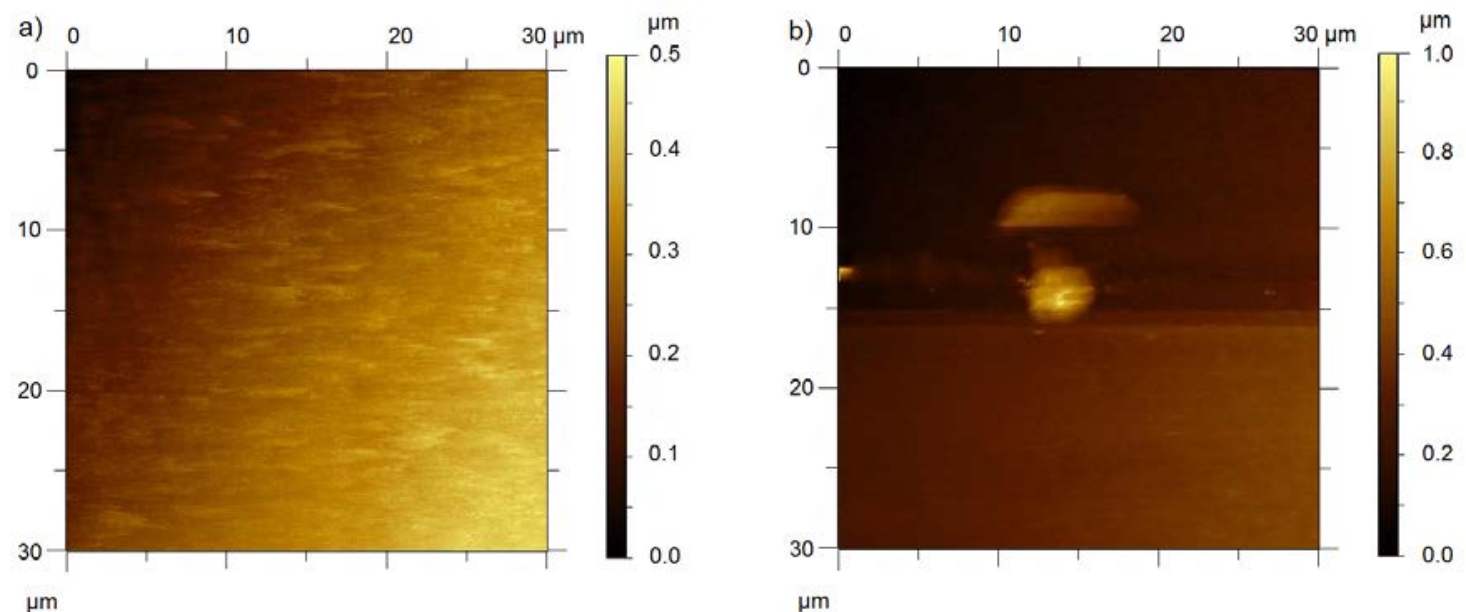

Figure 3. AFM topographies of the iron surface in $0.5 \mathrm{M} \mathrm{NaCl}+0.1 \mathrm{M} \mathrm{NaNO} 2$ at open circuit

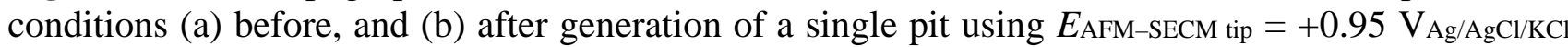
(sat.), followed by consecutive scans for iron(II) detection ( $E_{\mathrm{AFM}-\mathrm{SECM} \text { tip }}=+0.55 \mathrm{VAg} / \mathrm{AgCl} / \mathrm{KCl}$ (sat.)), and for oxygen reduction ( $E_{\mathrm{AFM}-\mathrm{SECM} \text { tip }}=-0.55 \mathrm{VAg} / \mathrm{AgCl} / \mathrm{KCl}$ (sat.) $)$ (not shown). Images (a) and (b) correspond to a scan area of $30 \times 30 \mu \mathrm{m}^{2}$. Scans for single corrosion pit generation and iron(II) detection were $3 \times 3$ $\mu \mathrm{m}^{2}$ in the center of image (a). Scan rate: $20 \mu \mathrm{m} \mathrm{s}^{-1}$. 


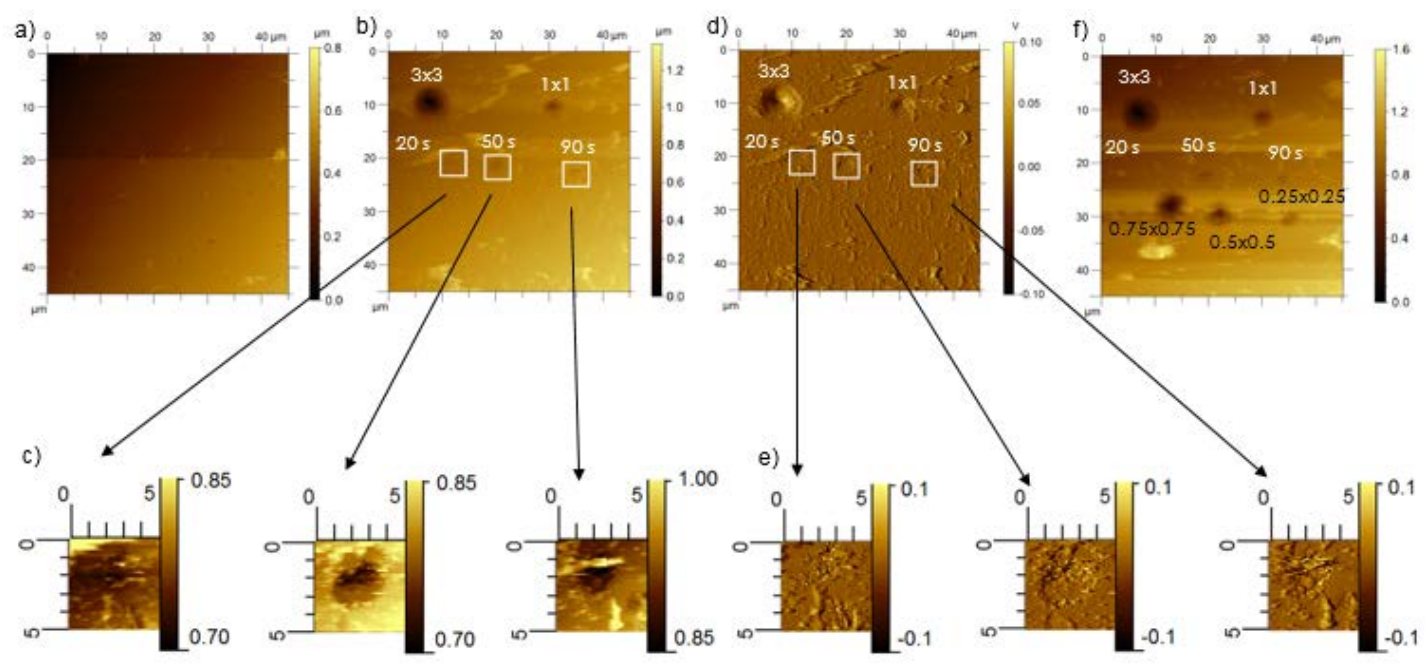

Figure 4. AFM (a-c,f) topographies and (d,e) deflection images of the iron surface in $0.5 \mathrm{M} \mathrm{NaCl}+$ $0.1 \mathrm{M} \mathrm{NaNO}_{2}$ at open circuit conditions (a) before and (b-f) after various localized attacks at several locations using either scanning or stationary modes. Dimensions of the scan for scanning mode are given in $\mu^{2}$. (c) and (e) Zoomed view of the three attacks performed using the stationary mode that are shown in the respective simultaneously acquired (b) topographic and (d) deflection data. (f) The iron surface after a second series of localized attacks performed using the scanning mode;

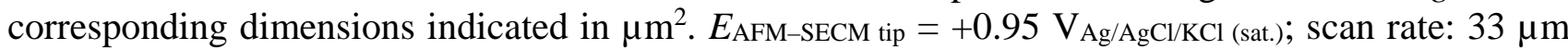
$\mathrm{s}^{-1}$.

a)

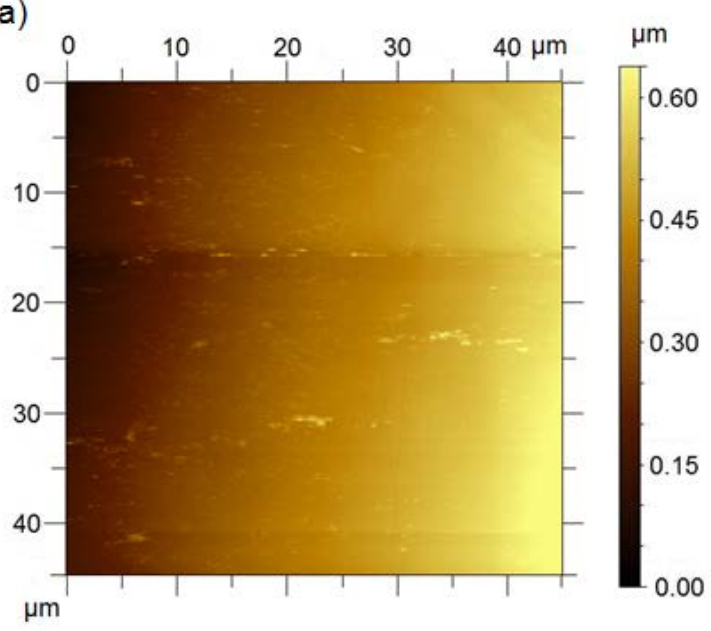

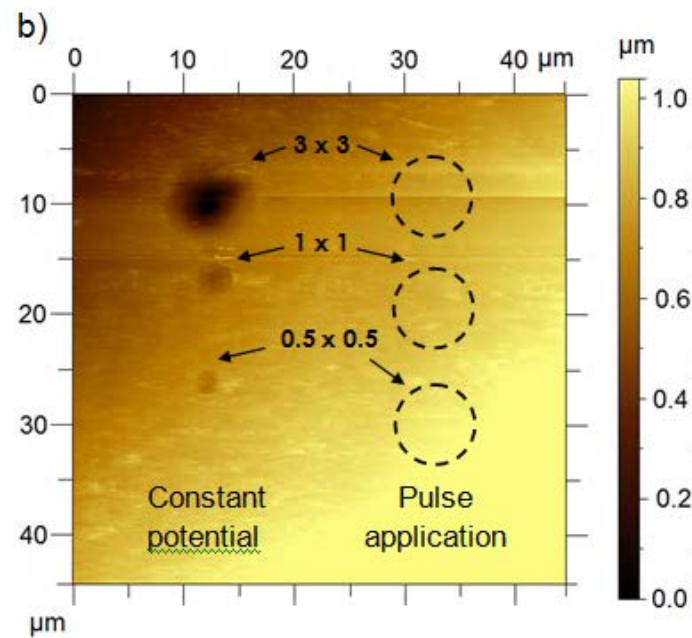

Figure 5. AFM topographies of the iron surface in $0.5 \mathrm{M} \mathrm{NaCl}+0.1 \mathrm{M} \mathrm{NaNO}_{2}$ at open circuit conditions (a) before and (b) after various localized attacks at several locations using scanning procedures with either (left) constant or (right) pulsed polarizations of the AFM-SECM tip. Approximate locations are indicated where the pulsed polarization was performed. Dimensions of the scans for the pit generation procedures are given in $\mu \mathrm{m}^{2}$. $E_{\mathrm{AFM}-\mathrm{SECM} \mathrm{tip}}=+0.95 \mathrm{~V}_{\mathrm{Ag} / \mathrm{AgCl} / \mathrm{KCl} \text { (sat.); }}$ AFM scan rate: $33 \mu \mathrm{m} \mathrm{s}^{-1}$. 


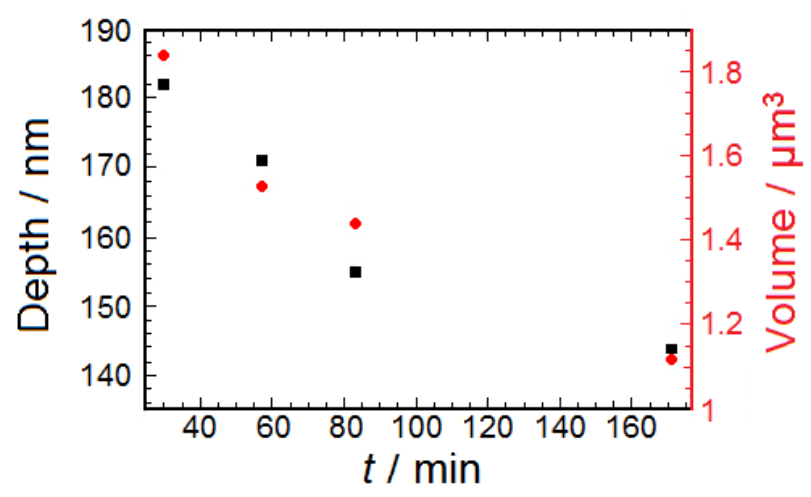

Figure 6. Time evolution of pit depth ( $\boldsymbol{\square})$ and volume $($ ) after induced pit formation determined from a sequence of AFM images.
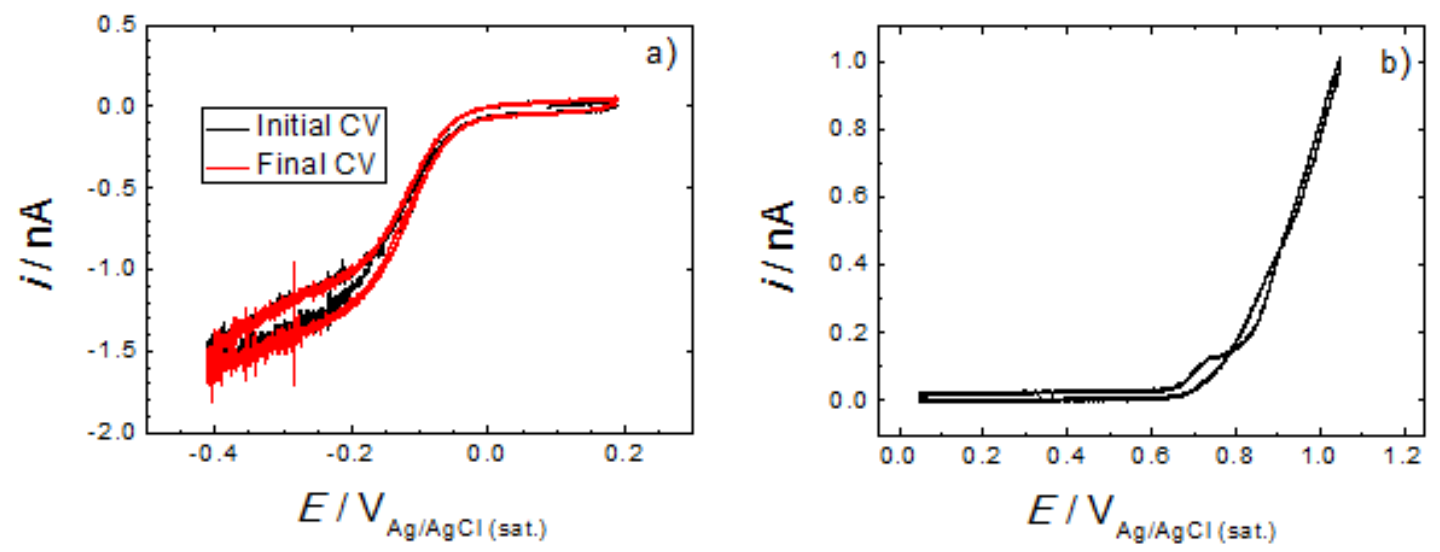

Figure 7. Cyclic voltammetry recorded at the AFM-SECM probe. (a) CVs recorded in $0.1 \mathrm{M} \mathrm{KCl}+10 \mathrm{mM}$ $\left[\mathrm{Ru}\left(\mathrm{NH}_{3}\right)_{6}\right] \mathrm{Cl}_{3}$ at the beginning and at the end of one experimental series consisting of 5 different pitting experiments. (b) $\mathrm{CV}$ recorded in $0.5 \mathrm{M} \mathrm{NaCl}+0.1 \mathrm{M} \mathrm{NaNO}_{2}$ prior to the initiation of the experimental series for (a). 